\title{
Epidemiology, clinical characteristics, and associations for symptom-based Rome IV functional dyspepsia in adults in the USA, Canada, and the UK: a cross-sectional population-based study
}

Imran Aziz, Olafur S Palsson, Hans Törnblom, Ami D Sperber, William E Whitehead, Magnus Simrén

\section{Summary}

Lancet Gastroenterol Hepatol 2018; 3: 252-62 Published Online January 29, 2018 http://dx.doi.org/10.1016/ S2468-1253(18)30003-7 See Comment page 217 Department of Internal Medicine and Clinical Nutrition, Institute of Medicine, Sahlgrenska Academy, University of Gothenburg, Gothenburg,

Sweden (I Aziz MD HTörnblom MD, Prof M Simrén MD); Center for Functional Gastrointestinal and Motility Disorders, University of North Carolina,

Chapel Hill, NC, USA

(Prof O S Palsson PsyD, Prof W E Whitehead PhD, Prof M Simrén); and Faculty of Health Sciences, Ben-Gurion

University of the Negev, Beer-Sheva, Israel (Prof A D Sperber MD)

Correspondence to: Dr Imran Aziz, Department of Internal Medicine and Clinical Nutrition, Institute of Medicine, Sahlgrenska Academy, University of Gothenburg, Gothenburg, 41345, Sweden imran.aziz@sth.nhs.uk

Background The population prevalence, clinical characteristics, and associations for Rome IV functional dyspepsia are not known. Following the publication of the Rome IV criteria for functional gastrointestinal disorders, we aimed to assess the prevalence, characteristics, and associations for symptom-based Rome IV functional dyspepsia in adults across the USA, Canada, and the UK.

Methods We sent an internet-based cross-sectional health survey to adults in the general population of three English-speaking countries: the USA, Canada, and the UK. We used quota-based sampling to generate demographically balanced and population-representative samples. Individuals were invited to complete an online questionnaire on general health, without mention that the purpose of this survey was to examine gastrointestinal symptoms. We excluded participants who failed two attention-test questions or were excessively inconsistent on the three gastrointestinal questions that were presented twice in the survey for this particular purpose. The survey enquired about demographics, health-care visits, medications, somatisation, quality of life, and symptom-based criteria for Rome IV functional dyspepsia as well as for irritable bowel syndrome (IBS) and functional heartburn. We made subsequent comparisons between participants with Rome IV functional dyspepsia and controls without dyspepsia. The primary objective was to identify participants who fulfilled symptom-based criteria for Rome IV functional dyspepsia and categorise them into postprandial distress syndrome, epigastric pain syndrome, or overlapping subtypes.

Findings 6300 general population adults completed the health survey; 2100 each from the USA, Canada, and the UK. 369 responses were deemed inconsistent, leaving data for 5931 adults. Rome IV functional dyspepsia was significantly more prevalent in the USA (232 [12\%] of 1949) than in Canada (167 [8\%] of 1988) and the UK (152 [8\%] of 1994; $\mathrm{p}<0.0001)$. The subtype distribution was $61 \%$ postprandial distress syndrome, $18 \%$ epigastric pain syndrome, and $21 \%$ overlapping variant with both syndromes; this pattern was similar across the countries. Participants with functional dyspepsia had significantly greater health impairment and health-care usage than those without dyspepsia. Participants with the overlapping variant showed greater somatisation and poorer quality-of-life scores than did individuals with either postprandial distress syndrome or epigastric pain syndrome alone. In multivariate analysis, independent factors associated with all functional dyspepsia subtypes included worsening quality of life and the presence of symptoms compatible with functional heartburn and IBS, with functional heartburn and IBS having the strongest association with overlapping postprandial distress syndrome and epigastric pain syndrome. Notably, somatisation showed a positive association with postprandial distress syndrome and the overlapping variant, and use of antidepressants showed a negative association with postprandial distress syndrome.

Interpretation Approximately $10 \%$ of the adult population fulfils symptom-based criteria for Rome IV functional dyspepsia and incurs considerable associated health impairment. The functional dyspepsia subtypes show differing associations, suggesting differences in pathophysiological processes or influences.

Funding The Rome Foundation, the US National Institute of Diabetes and Digestive and Kidney Diseases, the Swedish Medical Research Council, AFA Insurance, Ferring Pharmaceuticals, and the Faculty of Medicine, University of Gothenburg, Gothenburg, Sweden.

\section{Introduction}

Dyspepsia is a constellation of symptoms referable to the gastroduodenal region of the upper gastrointestinal tract. ${ }^{1}$ It has been reported to affect an estimated $21 \%$ of the general population, with prevalence varying from $29.5 \%$ when using broad historical definitions (ie, any upper gastrointestinal symptoms, including reflux), to
$7.6 \%$ when using the symptoms defined by the Rome III committee (ie, epigastric pain or burning [or both], postprandial fullness or early satiety [or both]). ${ }^{2}$ More than $70 \%$ of people with dyspepsia undergoing endoscopy will have no identifiable organic pathology to account for their symptoms and will be diagnosed as having functional dyspepsia, ${ }^{3}$ which can be further subgrouped 


\section{Research in context}

\section{Evidence before this study}

Functional dyspepsia is associated with overlapping functional gastrointestinal disorders, poor quality of life, and increased health-care usage. Although functional dyspepsia is perceived to be common, its prevalence has shown wide variations, even within similar geographical regions, due to differences in sampling methods and the criteria used to define its presence. We searched PubMed and our own files for articles relevant to functional dyspepsia published up to Sept 1, 2017, with the search terms "dyspepsia", "functional dyspepsia", "epigastric pain syndrome", and "postprandial distress syndrome", without a start date or language restrictions. We found no articles that assessed the prevalence of functional dyspepsia across multiple communities using any of the criteria proposed by the Rome Foundation, an international panel of experts working in the field of functional gastrointestinal disorders for almost 30 years. Furthermore, the association between somatisation and functional dyspepsia has been limited to a few studies that showed conflicting results. Following the publication of the Rome IV criteria for functional gastrointestinal disorders, we aimed to assess the prevalence, characteristics, and associations for symptom-based Rome IV functional dyspepsia in adults across the USA, Canada, and the UK.

\section{Added value of our study}

To our knowledge, we report for the first time that approximately $10 \%$ of the adult population from the USA,
Canada, and the UK fulfil the Rome IV symptom-based criteria for functional dyspepsia. We found that the highest prevalence is in the USA (12\%), compared with Canada (8\%) and the UK (8\%). The predominant functional dyspepsia subtype in each country is postprandial distress syndrome (about $60 \%$ ), followed by a relatively even split between epigastric pain syndrome and the overlapping variant. All functional dyspepsia subtypes had greater health impairment, including somatisation, than those without dyspepsia. We found that antidepressants might reduce the association with functional dyspepsia, in particular for the postprandial distress syndrome variant.

\section{Implications of all the available evidence}

Awareness of the epidemiological scale of Rome IV functional dyspepsia, and its detrimental effect on general health and wellbeing, will aid clinical service and research provision planning. This is pertinent given that current international guidelines acknowledge the lack of effective therapy for functional dyspepsia. Furthermore, current guidelines for dyspepsia largely focus on the epigastric pain variant and our study suggests that the predominant subtype is the postprandial distress syndrome variant, for which therapies are arguably even more unsatisfactory. into epigastric pain syndrome, postprandial distress syndrome, or the overlapping variant. ${ }^{4}$ Furthermore, functional dyspepsia represents a substantial health burden, being associated with increased health-care usage, $^{5,6}$ psychological disturbances, ${ }^{7-9}$ somatisation, ${ }^{8,10}$ impaired quality of life, ${ }^{11}$ and presence of overlapping functional gastrointestinal disorders such as irritable bowel syndrome (IBS) and heartburn. ${ }^{12,13}$

Limitations remain in research into functional dyspepsia. First, the prevalence of functional dyspepsia, as defined $\mathrm{b}$ y t he $\mathrm{R}$ ome $\mathrm{c}$ riteria, $\mathrm{h}$ as $\mathrm{n}$ ot $\mathrm{b}$ een simultaneously evaluated across multiple countries. ${ }^{2}$ Rather, previous studies using Rome I to III criteria have been limited to evaluations within single communities and, when pooled within the domains of their respective criteria, have shown wide prevalence ranges and substantial heterogeneity, with differences noted in sample size, symptom duration, and methods used to collect symptom data. ${ }^{2}$ Furthermore, within the context of Rome III functional dyspepsia, a meta-analysis found only seven studies, of which six were from Asia and one was from the USA, with prevalence ranging from $1 \cdot 8 \%$ to $17 \cdot 5 \%$; $^{2}$ clinical and epidemiological differences i n $\mathrm{f}$ unctional d yspepsia have been noted between Asia compared with Europe and North America. ${ }^{14}$ Second, an overarching perspective of the effects of functional dyspepsia on multiple health parameters has not been concurrently evaluated, but rather limited to comparisons against single or few health measurements. ${ }^{5-13}$ Third, data are conflicting with regard to functional dyspepsia and somatisation; one group suggested that the overlapping variant is associated with worse somatisation than postprandial distress syndrome or epigastric pain syndrome alone, ${ }^{8}$ but another did not replicate these findings. ${ }^{10}$

The publication of the Rome IV criteria for functional gastrointestinal disorders provides an opportunity to address these uncertainties in a systematic and homogeneous manner, using the most contemporaneous definition. ${ }^{15-17}$ We analysed data from a large cross-sectional population-based survey across three English-speaking countries with the aim to further understand the epidemiology and clinical associations of Rome IV functional dyspepsia and its subtypes, with participants without dyspepsia as comparative controls.

\section{Methods}

\section{Study design and participants}

We commissioned Qualtrics (Provo, UT, USA), a global market survey company, in 2015 to provide a nationally representative general population sample of adults from three English-speaking countries: the USA, Canada, and the UK. Quota-based sampling was used 

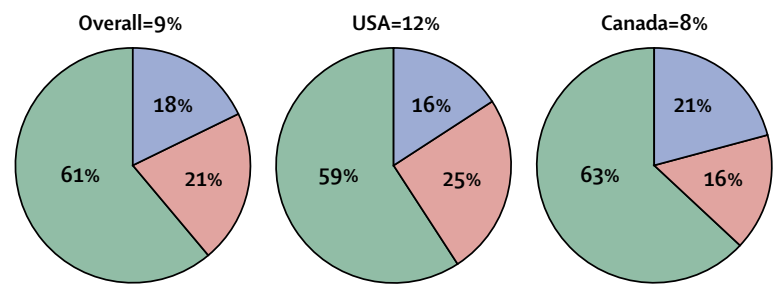

$\square$ Postprandial distress syndrome

$\square$ Epigastric pain syndrome

$\square$ Overlapping

Figure 1: Population prevalence of Rome IV functional dyspepsia and subtypes using symptom-based criteria

\begin{tabular}{|c|c|c|c|}
\hline & $\begin{array}{l}\text { No dyspepsia } \\
(n=5380)\end{array}$ & $\begin{array}{l}\text { Rome IV } \\
\text { functional } \\
\text { dyspepsia } \\
(n=551)\end{array}$ & p value \\
\hline \multicolumn{4}{|l|}{ Demographics } \\
\hline Age (years) & $47 \cdot 8(17 \cdot 2)$ & $43 \cdot 8(16 \cdot 2)$ & $<0.0001$ \\
\hline Female & $2587(48 \%)$ & $331(60 \%)$ & $<0.0001$ \\
\hline White & $3882(72 \%)$ & $389(71 \%)$ & 0.44 \\
\hline \multicolumn{4}{|l|}{ Relationship status } \\
\hline Single & $1591(30 \%)$ & $161(29 \%)$ & 0.90 \\
\hline Married or cohabiting & $2997(56 \%)$ & $305(55 \%)$ & 0.86 \\
\hline Divorced & $551(10 \%)$ & $61(11 \%)$ & $0 \cdot 54$ \\
\hline Widowed & $241(5 \%)$ & $24(4 \%)$ & 0.89 \\
\hline \multicolumn{4}{|l|}{ Symptom scores } \\
\hline PHQ-12 somatisation score & $4 \cdot 4(3 \cdot 5)$ & $8 \cdot 8(4.4)$ & $<0.0001$ \\
\hline Number of somatic symptoms & $3 \cdot 5(2 \cdot 5)$ & $6 \cdot 2(2 \cdot 6)$ & $<0.0001$ \\
\hline SF8-PCS & $49 \cdot 9(9 \cdot 3)$ & $41 \cdot 8(11 \cdot 3)$ & $<0.0001$ \\
\hline SF8-MCS & $49 \cdot 9(10 \cdot 3)$ & $40 \cdot 4(12 \cdot 7)$ & $<0.0001$ \\
\hline \multicolumn{4}{|l|}{ Overlapping FGIDs } \\
\hline IBS & $167(3 \%)$ & $174(32 \%)$ & $<0.0001$ \\
\hline Functional heartburn & $40(<1 \%)$ & $67(12 \%)$ & $<0.0001$ \\
\hline \multicolumn{4}{|l|}{ Health-care usage } \\
\hline $\begin{array}{l}\text { Seen doctor for gastrointestinal } \\
\text { health problems }\end{array}$ & $1131(21 \%)$ & $240(44 \%)$ & $<0.0001$ \\
\hline $\begin{array}{l}\text { More than once yearly } \\
\text { health-care visits }\end{array}$ & $3003(56 \%)$ & $404(73 \%)$ & $<0.0001$ \\
\hline \multicolumn{4}{|c|}{ Medication taken at least once a week } \\
\hline Antiemetic & $121(2 \%)$ & $84(15 \%)$ & $<0.0001$ \\
\hline Acid-suppressive medication & $905(17 \%)$ & $250(45 \%)$ & $<0.0001$ \\
\hline Analgesic & $1450(27 \%)$ & $287(52 \%)$ & $<0.0001$ \\
\hline Antidepressant & $653(12 \%)$ & $140(25 \%)$ & $<0.0001$ \\
\hline Any of the above medication & $2153(40 \%)$ & $395(72 \%)$ & $<0.0001$ \\
\hline
\end{tabular}

Data are mean (SD) or n (\%). PHQ-12=Patient Health Questionnaire-12 somatic symptom scale. SF8-PCS=short form-8 quality-of-life form-physical component score. SF8-MCS=SF8-mental component score. FGIDs=functional gastrointestina disorders. IBS=irritable bowel syndrome.

Table 1: Univariate analysis comparing participants without dyspepsia with those with Rome IV functional dyspepsia

to generate demographically balanced and populationrepresentative samples with regard to age, sex, and education. Extensive details regarding the geographical distribution (by state, province or territory, or government region) and demographic profile of participants, and how closely they resemble the general population, have been described elsewhere. ${ }^{18}$ Before data collection started, the study was reviewed by the Institutional Review Board (IRB) at the University of North Carolina (Chapel Hill, NC, USA), and deemed IRB exempt because all study participants were anonymous to the investigators. The study was done in accordance with the STROBE statement.

\section{Procedures}

Individuals were invited to complete an online questionnaire on general health, without mention that the purpose of this survey was to examine gastrointestinal symptoms. The comprehensive questionnaire collected information about demographic characteristics (age, gender, race, relationship status, and country of residence), medication (antiemetic, acid-suppressive medication, analgesics, and antidepressants), health-care visits, somatisation, ${ }^{19}$ quality of life, ${ }^{20}$ and the presence of Rome IV functional gastrointestinal disorders. ${ }^{17}$ Further details on the questionnaires have been described previously. ${ }^{18}$

Multiple quality assurance methods were built into the survey to minimise bias and poor quality reporting. ${ }^{18}$ These included the software detecting missing answers for any applicable question and ensuring participants completed these before being allowed to proceed. Furthermore, taking into consideration the possibility of inconsistent responders, we excluded participants who failed two attention-test questions or were excessively inconsistent on the three gastrointestinal questions that were presented twice in the survey for this particular purpose..$^{18}$

\section{Outcomes}

The primary outcome was to identify participants who fulfilled symptom-based criteria for Rome IV functional dyspepsia and to categorise them into postprandial distress syndrome, epigastric pain syndrome, or overlapping subtypes. ${ }^{21}$ We did not ask participants about previous endoscopies and, given the study design, could not perform endoscopies. The definition of epigastric pain syndrome was based on at least 1 day per week of bothersome epigastric pain, burning, or both. The definition of postprandial distress syndrome was based on at least 3 days per week of bothersome postprandial fullness, early satiety, or both. Those with the overlapping variant fulfilled criteria for both. Furthermore, the symptoms had to be present for the last 3 months with onset at least 6 months previously. ${ }^{21}$ All individuals in the population who did not meet Rome IV symptom-based criteria for functional dyspepsia were used as control participants in the analyses.

In those with and without functional dyspepsia, we also identified the proportion of participants who had symptoms compatible with functional heartburn and IBS in accordance with the Rome IV criteria. ${ }^{22,23}$ 


\section{Statistical analysis}

We did statistical analyses using SPSS version 21.0, with $p$ values of 0.05 or less deemed to be significant. There were no missing data because the online questionnaire required participants to complete each applicable question before being allowed to move on to the next step. We determined the prevalence and characteristics of the overall population, those with and without functional dyspepsia, and the functional dyspepsia subtypes. We summarised categorical variables by descriptive statistics, including total numbers and percentages, with comparisons between groups performed using the $\chi^{2}$ test. We summarised continuous variables by mean and $\mathrm{SD}$, with differences b etween $\mathrm{t}$ wo i ndependent $\mathrm{g}$ roups compared using the unpaired Student's $t$ test. For comparison of continuous data between multiple groups we used the one-way analysis of variance. We did post-hoc testing using Bonferroni correction.

We did binary logistic regression to identify independent associations for functional dyspepsia (and its individual subtypes) against controls without dyspepsia. All independent variables were considered for analysis in the regression model, but before their entry we assessed for multicollinearity using linear regression and checked for a variance inflation factor cutoff of gr eater than 3 . Th is revealed a strong multicollinearity between number of somatic symptoms and Patient Health Questionnaire (PHQ-12) somatisation score (variance inflation factor $>10$ ), and as a result, the number of somatic symptoms was subsequently omitted from the binary logistic regression model. Otherwise, the following independent predictor variables that might be associated with functional dyspepsia were entered in one block: age, female sex, white race, relationship status, country of residence, health-care visits, medication, PHQ-12 somatisation scores, mental and physical quality-of-life scores, and presence of symptoms compatible with IBS and functional heartburn. These data were presented using adjusted odds ratios (AORs) and 95\% CIs.

\section{Role of the funding source}

The funders had no role in study design, data collection, data analysis, data interpretation, or writing of the report. The corresponding author had access to all data in the study and had the final $r$ esponsibility f or the decision to submit for publication.

\section{Results}

The survey was completed by 6300 individuals: 2100 from each of the three countries. 369 (5.9\%) inconsistent responders were excluded from the analysis, leaving responses from 5931 individuals in the analysis dataset: 1949 from the USA, 1988 from Canada, and 1994 from the UK. Mean age was 47.4 years (SD 17.1), 2918 (49\%) participants were female, and 4271 (72\%) were white. Further characteristics have been described elsewhere. ${ }^{18}$

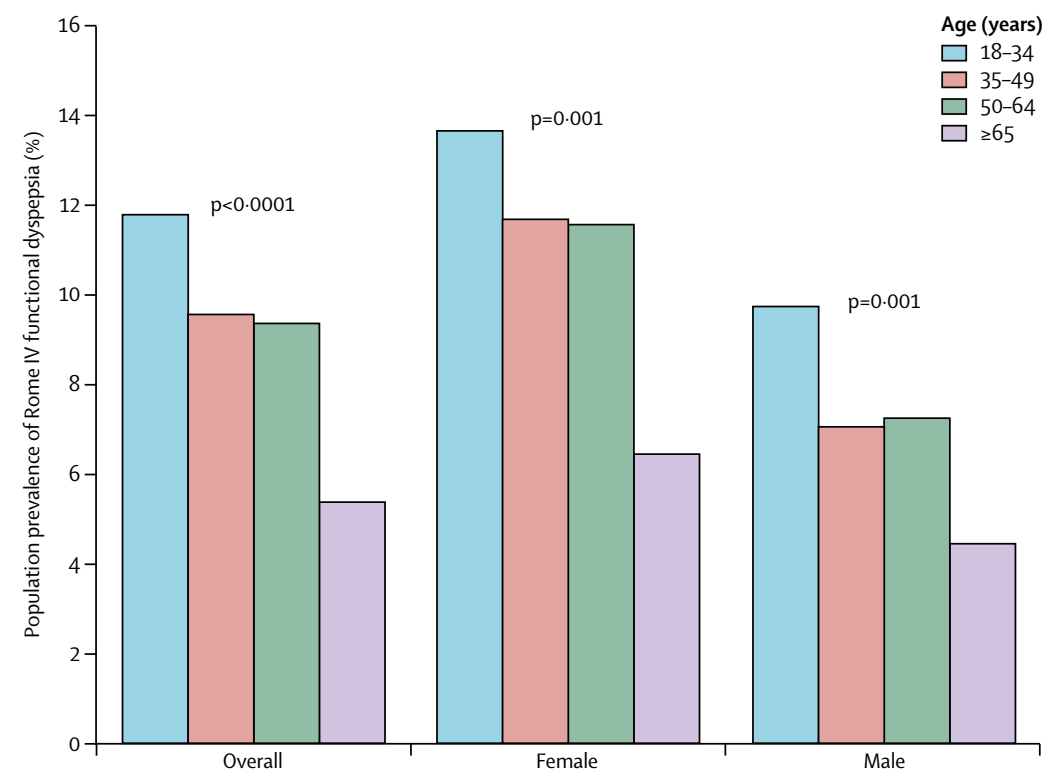

Figure 2: Prevalence of Rome IV functional dyspepsia according to sex and age group

To assess the prevalence of Rome IV functional dyspepsia and its subtypes, we examined the 5931 participants available for evaluation. 5380 (91\%) did not meet symptom-based criteria for Rome IV functional dyspepsia and were classed as controls. The remaining $551(9 \%)$ participants within the population fulfilled symptom-based criteria for Rome IV functional dyspepsia, with 339 (6\%) having postprandial distress syndrome, 97 (2\%) having epigastric pain syndrome, and $115(2 \%)$ having the overlapping variant.

Rome IV functional dyspepsia was significantly more prevalent in the USA ( $\mathrm{n}=232$ [12\%] of 1949) than in Canada (167 [8\%] of 1988) and the UK (152 [8\%] of 1994; $\mathrm{p}<0 \cdot 0001)$. Furthermore, the USA had a higher prevalence of postprandial distress syndrome (137 [7\%]) than Canada (106 [5\%]) and the UK (96 [5\%]; $\mathrm{p}=0.007)$. The prevalence of epigastric pain syndrome in the USA (36 [2\%]), Canada (35 [2\%]), and the UK (26 [1\%]) was similar $(\mathrm{p}=0 \cdot 35)$. However, the USA had an increased prevalence of the overlapping variant (59 [3\%]) compared with Canada (26 [1\%]) and the UK (30 [2\%]; p<0 0001).

Among participants with Rome IV functional dyspepsia, the distribution of subtypes was similar in the combined population and across all three countries; overall, postprandial distress syndrome was the most common subtype, accounting for $61 \%$ of all functional dyspepsia, with epigastric pain syndrome representing $18 \%$, and the overlapping variant representing $21 \%$ (figure 1).

We compared participants with Rome IV functional dyspepsia with non-dyspeptic controls (table 1). Participants with functional dyspepsia were significantly younger and predominantly female compared with controls, but a similar proportion were white and 
PDS ( $n=339) \quad$ EPS ( $n=97) \quad$ Overlapping PDS p value and EPS ( $n=115)$

\begin{tabular}{|c|c|c|c|c|}
\hline \multicolumn{5}{|l|}{ Demographics } \\
\hline Age (years) & $45(16 \cdot 8)$ & $42 \cdot 5(15 \cdot 6)$ & $41 \cdot 2(14 \cdot 4)$ & 0.068 \\
\hline Female & $211(62 \%)$ & $52(54 \%)$ & $68(59 \%)$ & 0.302 \\
\hline White & $242(71 \%)$ & $70(72 \%)$ & $77(67 \%)$ & 0.622 \\
\hline \multicolumn{5}{|l|}{ Relationship status } \\
\hline Single & $91(27 \%)$ & $36(37 \%)$ & $34(30 \%)$ & 0.146 \\
\hline Married or cohabiting & $198(58 \%)$ & $47(49 \%)$ & $60(52 \%)$ & 0.164 \\
\hline Divorced & $35(10 \%)$ & $11(11 \%)$ & $15(13 \%)$ & 0.721 \\
\hline Widowed & $15(4 \%)$ & $3(3 \%)$ & $6(5 \%)$ & 0.748 \\
\hline \multicolumn{5}{|l|}{ Symptom scores } \\
\hline PHQ-12 somatisation score & $8 \cdot 0(4 \cdot 0)$ & $8 \cdot 5(4 \cdot 3)$ & $11 \cdot 3(4 \cdot 7)$ & $<0.0001^{*} \dagger$ \\
\hline Number of somatic symptoms & $5.8(2.5)$ & $6 \cdot 1(2 \cdot 6)$ & $7 \cdot 5(2.5)$ & $<0.0001^{*} \dagger$ \\
\hline SF8-PCS & $43 \cdot 8(11 \cdot 2)$ & $40 \cdot 9(11 \cdot 2)$ & $37(10 \cdot 1)$ & $<0.0001^{*} \dagger$ \\
\hline SF8-MCS & $41 \cdot 5(12 \cdot 8)$ & $40 \cdot 0(13 \cdot 3)$ & $37 \cdot 6(11 \cdot 3)$ & $0.016^{*}$ \\
\hline \multicolumn{5}{|l|}{ Overlapping FGIDs } \\
\hline IBS & $50(15 \%)$ & $41(42 \%)$ & $83(72 \%)$ & $<0.0001^{*} \dagger \ddagger$ \\
\hline Functional heartburn & $21(6 \%)$ & $11(11 \%)$ & $35(30 \%)$ & $<0.0001^{*} \dagger$ \\
\hline \multicolumn{5}{|l|}{ Health-care usage } \\
\hline $\begin{array}{l}\text { Seen doctor for gastrointestinal } \\
\text { health problems }\end{array}$ & $118(35 \%)$ & $53(55 \%)$ & $69(60 \%)$ & $<0.0001^{*} \ddagger$ \\
\hline $\begin{array}{l}\text { More than once yearly health-care } \\
\text { visits }\end{array}$ & $241(71 \%)$ & $70(72 \%)$ & $93(81 \%)$ & $0 \cdot 118^{*}$ \\
\hline \multicolumn{5}{|c|}{ Medication taken at least once a week } \\
\hline Antiemetic & $39(12 \%)$ & $13(13 \%)$ & $32(28 \%)$ & $<0.0001^{*} \dagger$ \\
\hline Acid-suppressing drug & $122(36 \%)$ & $54(56 \%)$ & $74(64 \%)$ & $<0.0001^{*} \ddagger$ \\
\hline Analgesic & $156(46 \%)$ & $58(60 \%)$ & $73(64 \%)$ & $0.0001^{*} \ddagger$ \\
\hline Antidepressant & $71(21 \%)$ & $26(27 \%)$ & $43(37 \%)$ & $0.002^{*}$ \\
\hline Any of the above medication & $218(64 \%)$ & $80(83 \%)$ & $97(84 \%)$ & $<0.0001^{*} \ddagger$ \\
\hline
\end{tabular}

Data are mean (SD) or $n$ (\%). PDS=postprandial distress syndrome. EPS=epigastric pain syndrome. PHQ-12=Patient Health Questionnaire-12 somatic symptom scale. SF8-PCS=short form-8 quality-of-life form-physical component score. SF8-MCS=SF8-mental component score. FGIDs=functional gastrointestinal disorders. *Indicates the overlap group is significantly different compared with the PDS group. †Indicates the overlap group is significantly different compared with the EPS group. ¥Indicates EPS group is significantly different compared with the PDS group. Presented $p$ values are across the groups.

Table 2: Comparison between Rome IV functional dyspepsia subtypes

relationship statuses were also similar. The prevalence of functional dyspepsia was significantly higher between the ages of 18 and 34 years (200 [12\%] of 1693) than in the age groups $35-49$ years (131 [10\%] of 1365) and 50-64 years (154 [9\%] of 1642), with the lowest prevalence of functional dyspepsia being seen in individuals older than 65 years (66 [5\%] of 1226; $\mathrm{p}<0 \cdot 0001)$. This pattern was seen across both sexes (figure 2). Female individuals had a significantly greater prevalence of functional dyspepsia than their male counterparts across all age groups, except for in those older than 65 years, for whom there was no statistical difference $(p=0 \cdot 12)$.

Individuals with functional dyspepsia had significantly higher PHQ-12 somatisation scores and number of somatic symptoms, worse mental and physical quality of life, a higher prevalence of IBS, and were more likely to have symptoms compatible with functional heartburn, compared with controls (table 1). Participants with functional dyspepsia reported greater health-care usage compared with controls, with on average $73 \%$ seeking health care more than once yearly, $44 \%$ consulting for gastrointestinal-related problems, and $72 \%$ taking dyspepsia-relevant medications (ie, antiemetics, acid-suppressive medications, analgesics, or antidepressants).

We did a comparison of the subtypes of Rome IV functional dyspepsia (table 2). There were no differences in age, sex, white race, and relationship status between the subgroups. However, participants with the overlapping variant showed greater somatisation and poorer quality-of-life scores than individuals with either syndrome alone. Furthermore, we analysed PHQ-12 and quality-of-life patterns according to country and health-care usage, and found that the overlapping group generally had greater somatisation (figure 3) and worse quality-of-life scores (figure 4) than either syndrome alone.

The prevalence of IBS was also significantly higher among those with overlapping postprandial distress syndrome and epigastric pain syndrome $(72 \%)$ than in those with epigastric pain syndrome alone $(42 \%)$ and was lowest in those with postprandial distress syndrome alone (15\%). Furthermore, the prevalence of symptoms compatible with functional heartburn was also highest among the overlapping group (30\%), which was significantly higher than the epigastric pain syndrome $(11 \%)$ and postprandial distress syndrome alone groups $(6 \%)$. Those in the overlapping group and epigastric pain syndrome alone group had significantly more health-care visits, gastrointestinal consultations, and dyspepsia-relevant medication than those in the postprandial distress syndrome alone group (table 2).

We also examined associations with Rome IV functional dyspepsia and subtypes (table 3). Younger age was associated with functional dyspepsia, epigastric pain syndrome, and the overlapping variant, but not with postprandial distress syndrome. Additionally, being from the USA was associated with functional dyspepsia, postprandial distress syndrome, and the overlapping variant, but not with epigastric pain syndrome, compared with those from the UK and Canada. Neither sex or relationship status had any significant association with functional dyspepsia or the subtypes.

The presence of symptoms compatible with functional heartburn and IBS was associated with all dyspepsia types, with the strongest association seen in those with overlapping postprandial distress syndrome and epigastric pain syndrome. Similarly, worse physical and mental quality-of-life scores were independently associated with functional dyspepsia and its subtypes. However, increasing somatisation was associated with functional dyspepsia, postprandial distress syndrome, and the overlapping group, but not with the epigastric pain syndrome group (table 3 ). 
The use of acid-suppressive medications was associated with functional dyspepsia and its subtypes. Antiemetic use was also associated with functional dyspepsia, postprandial distress syndrome, and the overlapping variant, but not with epigastric pain syndrome. Notably, the use of antidepressants had a negative association with functional dyspepsia (AOR 0.63, 95\% CI 0.47-0.83; $\mathrm{p}=0.001)$, and in particular with postprandial distress syndrome $(0 \cdot 61,0 \cdot 44-0 \cdot 85 ; \mathrm{p}=0 \cdot 003)$. Antidepressants might be negatively associated with epigastric pain syndrome $(0 \cdot 57,0 \cdot 31-1 \cdot 03 ; \mathrm{p}=0 \cdot 062)$ but this association was not significant a nd $\mathrm{t}$ his $\mathrm{w}$ as $\mathrm{n}$ ot $\mathrm{s}$ een $\mathrm{f}$ or $\mathrm{t}$ he overlapping group $(0 \cdot 72 ; 0 \cdot 39-1 \cdot 33 ; \mathrm{p}=0 \cdot 299)$. There was no independent association between the number of gastroenterology consultations or more than once yearly health-care visits and functional dyspepsia or its subtypes.

\section{Discussion}

To our knowledge, this is the first study to systematically evaluate the population prevalence of functional dyspepsia across multiple countries using the Rome criteria. We used the Rome IV iteration of the diagnostic criteria, which adds further to the novelty of this work. Furthermore, the study provides a comprehensive clinical and phenotypic profile o f i ndividuals w ith $\mathrm{R}$ ome I V functional dyspepsia, and identifies i ndependently associated factors. This provides a platform for understanding the current magnitude of functional dyspepsia, differences between the subtypes, and prioritising future research efforts.

We have shown that approximately $10 \%$ of the adult population fulfil Rome IV diagnostic criteria for functional dyspepsia, with postprandial distress syndrome representing approximately $60 \%$ of cases, followed by a relatively even split between epigastric pain syndrome and the overlapping variant. A higher prevalence of functional dyspepsia was found in the USA, compared with Canada and the UK, which remained significant after adjusting for all variables within the logistic regression model. Reasons for this difference could not be explored within this dataset but possible explanations include genetic, cultural, socioeconomic, dietary, and environmental factors. A systematic review ${ }^{2}$ has noted that risk factors for uninvestigated dyspepsia include smoking, non-steroidal anti-inflammatory drug usage, and $H$ elicobacter pylori status; however, these associations are modest and their overall importance in the cause of symptoms has been questioned. Some data also suggests that postprandial distress syndrome could be linked with duodenal eosinophilia, increased intestinal permeability, and neural changes, suggesting a potential infective or allergic component to this dyspeptic subtype. ${ }^{24}$ In line with these observations, data from a UK-based primary-care registry noted that individuals with functional dyspepsia had an increased prevalence of atopic disorders such as asthma. ${ }^{25}$ However, these novel

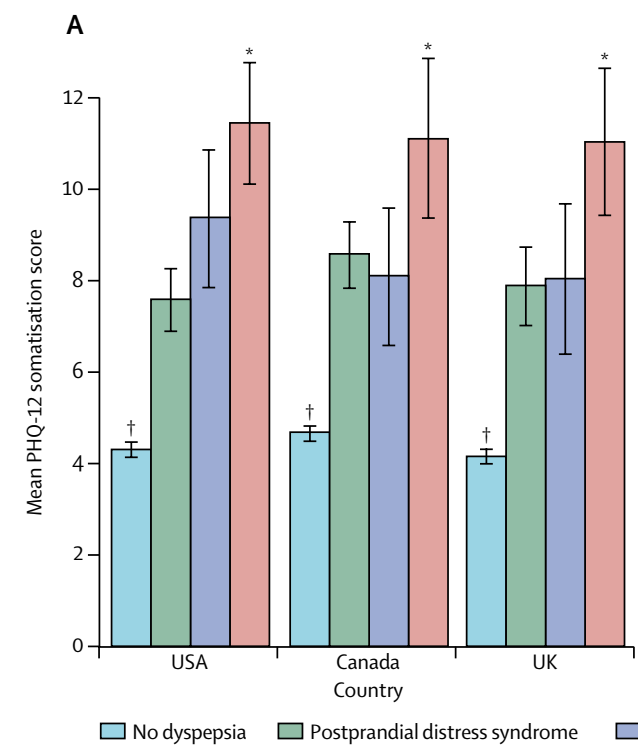

B

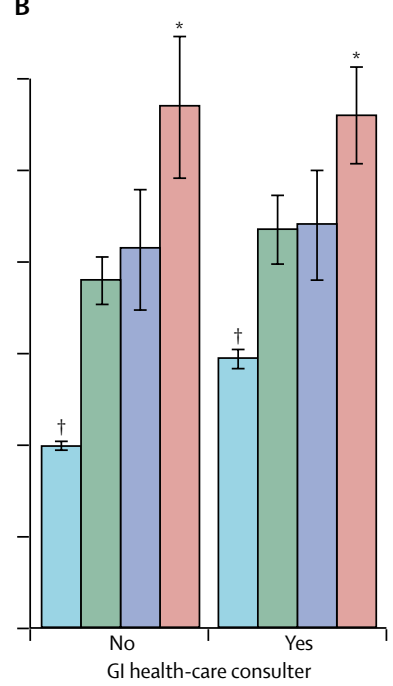

Figure 3: PHQ-12 somatisation scores in Rome IV functional dyspepsia subtypes according to (A) country and (B) health-care use

Error bars represent 95\% Cls. PHQ-12=Patient Health Questionnaire-12 somatic symptom scale. Gl=gastrointestinal. *Overlapping groups had significantly worse scores than postprandial distress syndrome and epigastric pain syndrome. †All functional dyspepsia subtypes had significantly worse somatisation scores than controls without dyspepsia $(p<0.0001)$. For all $p$ values, see appendix.

findings require replication and future studies might benefit from tracking how the prevalence of asthma correlates with functional dyspepsia, and in particular its postprandial distress syndrome subtype, among countries.

We showed that participants with Rome IV functional dyspepsia had greater health impairment and health-care usage that non-dyspeptic controls. Furthermore, we showed differences in clinical characteristics between subtypes of functional dyspepsia. Those with the overlapping variant incurred the greatest health impairment, as reflected by more frequent use of dyspepsia-relevant medications, increased prevalence of symptoms compatible with functional heartburn and IBS, poorer quality of life, and more severe somatisation. Increased somatisation is of particular interest because secondary-care studies ${ }^{8,10}$ have shown conflicting data with regard to somatisation in functional dyspepsia. An initial study ${ }^{8}$ from Taiwan suggested that individuals with the overlapping variant had greater somatisation compared with either syndrome alone, whereas, a study ${ }^{10}$ in Canadian patients did not find any significant differences. The study in Canadian patients, however, was limited to relatively small sample sizes (11 participants with epigastric pain syndrome and 33 participants with the overlapping variant, compared with 335 participants with postprandial distress syndrome). We believe our findings help clarify the uncertainty because we analysed data from a large sample, while also showing similar results irrespective of country or gastrointestinal consultation pattern. 


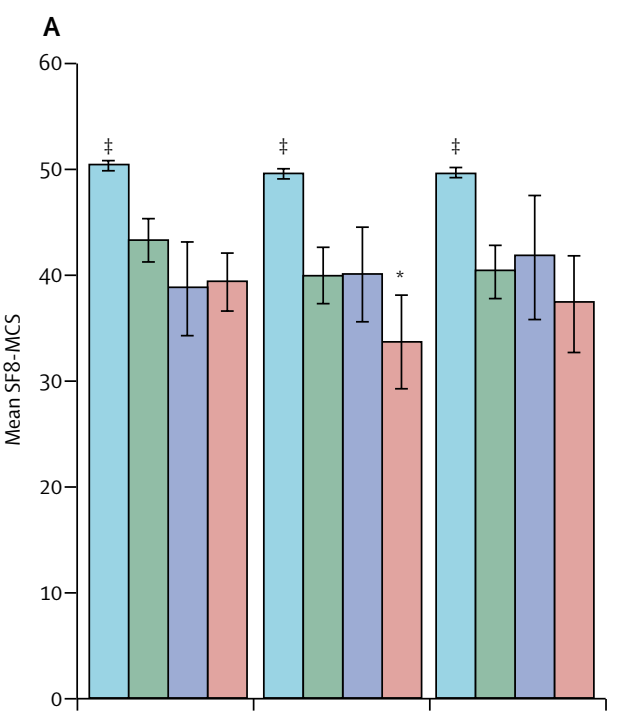

B
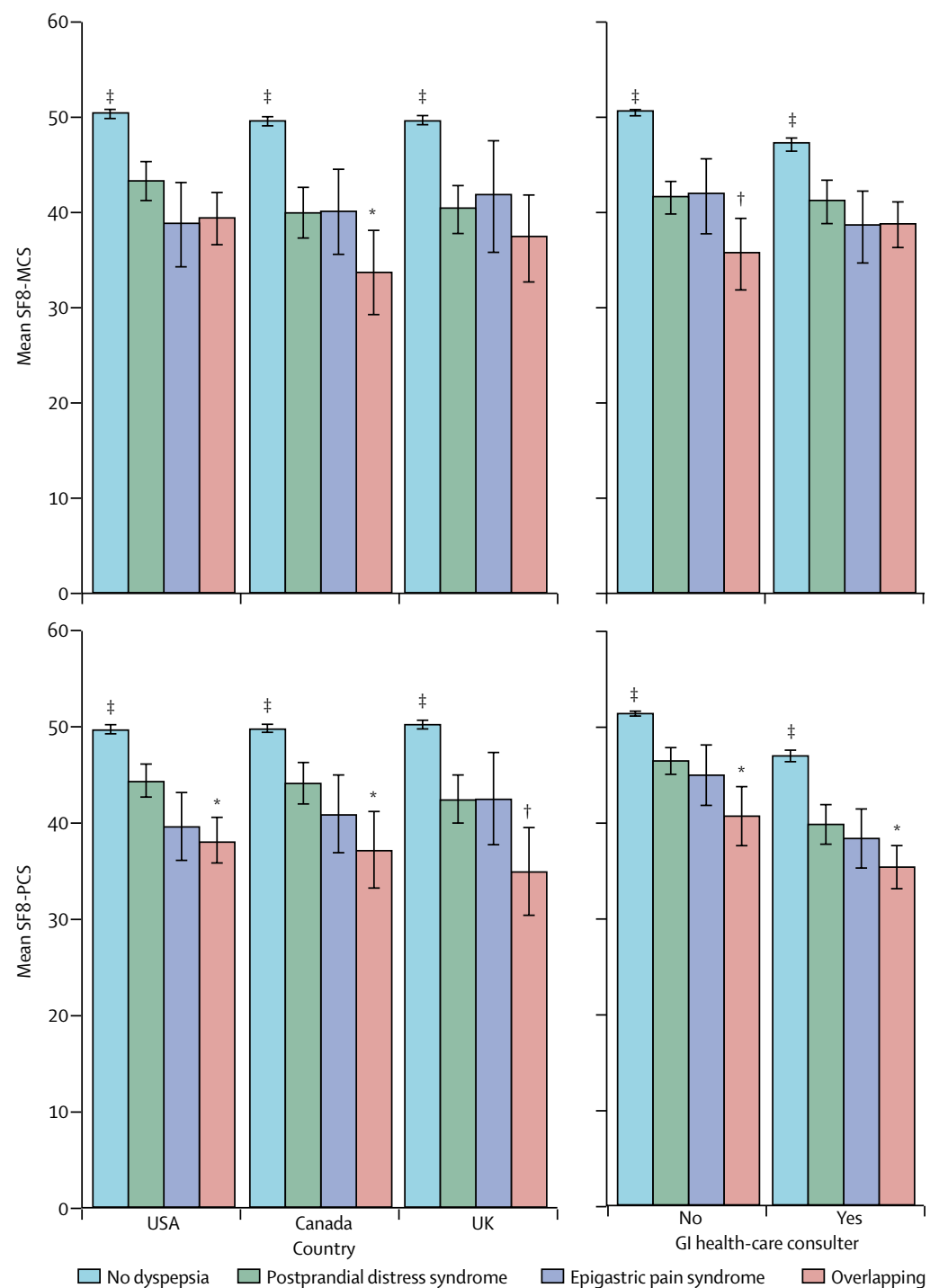

Figure 4: Quality-of-life scores in Rome IV functional dyspepsia subtypes according to (A) country and (B) health-care use

Error bars represent 95\% Cls. SF8-PCS=short component score. SF8-MCS= SF8-mental component score. $\mathrm{Gl}=$ gastrointestinal. * Overlapping groups had significantly worse scores than the postprandial distress syndrome group but not the epigastric pain syndrome group. †Overlapping groups had significantly worse scores than the postprandial distress syndrome group and the epigastric pain syndrome group. $¥$ All functional dyspepsia subtypes had significantly worse quality-of-life scores than controls without dyspepsia $(p<0.0001)$. For all p values, see appendix. in accordance with our findings are most commonly reported by those with the overlapping variant.

We explored independent factors associated with Rome IV functional dyspepsia and its subtypes. Common associations across all entities include presence of symptoms compatible with functional heartburn and IBS, worse mental and physical quality of life, and use of acid-suppressive medications. Previous longitudinal follow-up studies have noted that poor quality of life at baseline can lead to subsequent development of functional dyspepsia, ${ }^{33}$ and this is in line with our observations. The presence of other functional gastrointestinal disorders increasing the risk of functional dyspepsia has also been noted previously; ${ }^{12,13}$ however, we emphasised the strength of this association according to subtype. We showed that symptoms compatible with IBS or functional heartburn exert an incremental link towards postprandial distress syndrome, then epigastric pain syndrome, with the strongest association with overlapping postprandial distress and epigastric pain syndromes. This might be due to the overlap variant generally endorsing more symptoms although the difference seen between epigastric pain syndrome and postprandial distress syndrome alone might suggest different pathophysiological processes or influences. A potential hypothesis is that visceral hypersensitivity could be the predominant factor driving epigastric pain syndrome-which could explain the increased association with other functional gastrointestinal disorders seen in this group-whereas disturbed gastric accommodation and gastric emptying might drive postprandial distress syndrome. However, studies evaluating the role of these putative pathophysiological mechanisms among functional dyspepsia subtypes have shown conflicting results. ${ }^{34-38}$

We noted differences in the relationship of somatisation and use of antidepressants on functional dyspepsia. Previous studies have shown psychological indices such as anxiety, depression, somatisation, and sleep disturbances to predispose towards the development of postprandial distress syndrome but not epigastric pain syndrome..$^{7-9}$ We also found that somatisation was an independent factor positively associated with postprandial distress syndrome and the overlapping variant, but not for epigastric pain syndrome. Furthermore, our study provides novel supporting implications that use of antidepressants might independently reduce the link with functional dyspepsia, in particular for postprandial distress syndrome. We speculate that this association might be through antidepressants downregulating the dysfunctional brain-gut axis, via central inhibitory effects and peripheral mediating effects of increasing gastric accommodation, enhancing preprandial gastric relaxation, and altering gastric emptying rate. ${ }^{39-43}$ Our findings will hopefully stimulate further translational research efforts as,

although a systematic review and meta-analysis ${ }^{44}$ found
Previous studies have noted a discrepancy in functional dyspepsia subtype patterns in the general population compared with secondary care., ${ }^{96-30}$ Whereas the postprandial distress syndrome variant prevails in the community, the overlapping variant is the most commonly encountered subtype in hospital settings..$^{926-30}$ The reasons for this have not been determined, but factors that prompt specialist-care referrals for dyspepsia include greater symptom severity and coexisting functional gastrointestinal disorders, ${ }^{31,32}$ which 


\begin{tabular}{|c|c|c|c|c|c|c|c|c|}
\hline & \multicolumn{2}{|c|}{ Functional dyspepsia } & \multicolumn{2}{|l|}{ PDS } & \multicolumn{2}{|l|}{ EPS } & \multicolumn{2}{|c|}{ Overlapping PDS and EPS } \\
\hline & AOR $(95 \% \mathrm{Cl})$ & $p$ value & AOR $(95 \% \mathrm{Cl})$ & $p$ value & AOR $(95 \% \mathrm{Cl})$ & p value & AOR $(95 \% \mathrm{Cl})$ & $\mathrm{p}$ value \\
\hline Decreasing age & $1.01(1.00-1.02)$ & 0.027 & $1.00(0.99-1.01)$ & 0.444 & $1.02(1.00-1.04)$ & 0.017 & $1.03(1.01-1.05)$ & 0.017 \\
\hline Female & $1.03(0.83-1.27)$ & 0.809 & $1.15(0.89-1.48)$ & 0.275 & $0.79(0.50-1.25)$ & 0.312 & $0.94(0.55-1.58)$ & 0.801 \\
\hline White & $0.99(0.79-1.25)$ & 0.948 & $0.94(0.72-1.23)$ & 0.661 & $1.17(0.70-1.96)$ & 0.548 & $1.18(0.67-2 \cdot 10)$ & 0.567 \\
\hline \multicolumn{9}{|l|}{ Relationship status } \\
\hline Single & 1 & .. & 1 & .. & 1 & .. & 1 & .. \\
\hline Married & $0.97(0.76-1.25)$ & 0.833 & $1.11(0.82-1.48)$ & 0.490 & $0.66(0.39-1.10)$ & 0.112 & $0.85(0.46-1.55)$ & 0.590 \\
\hline Divorced & $0.96(0.65-1.41)$ & $0 \cdot 816$ & $1.01(0.64-1.59)$ & 0.983 & $0.72(0.32-1.63)$ & 0.427 & $1.43(0.59-3.45)$ & $0 \cdot 431$ \\
\hline Widowed & $1.17(0.67-2.06)$ & 0.575 & $1.13(0.59-2.17)$ & 0.711 & $0.55(0.13-2 \cdot 26)$ & 0.405 & $1.39(0.35-5 \cdot 46)$ & 0.640 \\
\hline \multicolumn{9}{|l|}{ Location } \\
\hline USA & 1 & .. & 1 & .. & 1 & .. & 1 &.. \\
\hline Canada & $0.65(0.51-0.83)$ & 0.001 & $0.68(0.51-0.90)$ & 0.006 & $0.75(0.44-1.26)$ & $0 \cdot 274$ & $0.35(0.19-0.66)$ & 0.001 \\
\hline UK & $0.65(0.51-0.84)$ & 0.001 & $0.68(0.51-0.91)$ & 0.009 & $0.59(0.33-1.05)$ & 0.071 & $0.55(0.30-1.00)$ & 0.051 \\
\hline \multicolumn{9}{|l|}{ Health-care usage } \\
\hline $\begin{array}{l}\text { Gastroenterology } \\
\text { consultations }\end{array}$ & $1.19(0.95-1.50)$ & $0 \cdot 138$ & $1.09(0.83-1.42)$ & 0.550 & $1.63(0.99-2.69)$ & 0.054 & $1.09(0.63-1.88)$ & 0.768 \\
\hline $\begin{array}{l}>1 \text { health-care visits } \\
\text { per year }\end{array}$ & $1 \cdot 11(0.87-1 \cdot 40)$ & 0.403 & $1 \cdot 19(0 \cdot 91-1 \cdot 58)$ & 0.192 & $0.95(0.56-1.60)$ & 0.833 & $1.25(0.67-2.33)$ & 0.488 \\
\hline \multicolumn{9}{|l|}{ Overlapping FGIDs } \\
\hline Functional heartburn & $3 \cdot 50(2 \cdot 14-5 \cdot 74)$ & $<0.0001$ & $2 \cdot 46(1 \cdot 30-4 \cdot 65)$ & 0.006 & $3 \cdot 18(1 \cdot 31-7 \cdot 71)$ & 0.011 & $7.05(3.30-15.04)$ & $<0.0001$ \\
\hline $\begin{array}{l}\text { Irritable bowel } \\
\text { syndrome }\end{array}$ & $3 \cdot 79(2 \cdot 83-5 \cdot 07)$ & $<0.0001$ & $1.63(1.09-2 \cdot 43)$ & 0.017 & $6.43(3.76-11.00)$ & $<0.0001$ & $17 \cdot 09(9 \cdot 80-29 \cdot 81)$ & $<0.0001$ \\
\hline \multicolumn{9}{|l|}{ Symptom scores } \\
\hline SF8-PCS & $1.03(1.02-1.04)$ & $<0.0001$ & $1.02(1.01-1.04)$ & $<0.0001$ & $1.04(1.01-1.06)$ & 0.004 & $1.03(1.01-1.06)$ & 0.017 \\
\hline SF8-MCS & $1.04(1.03-1.05)$ & $<0.0001$ & $1.04(1.03-1.05)$ & $<0.0001$ & $1.03(1.01-1.06)$ & 0.002 & $1.03(1.01-1.06)$ & 0.006 \\
\hline PHQ-12 somatisation & $1 \cdot 12(1 \cdot 10-1 \cdot 16)$ & $<0.0001$ & $1.12(1.08-1 \cdot 16)$ & $<0.0001$ & $1.04(0.97-1 \cdot 12)$ & 0.235 & $1.14(1.07-1.22)$ & $<0.0001$ \\
\hline \multicolumn{9}{|c|}{ Medication taken at least once a week } \\
\hline Antiemetic & $1.60(1.10-2 \cdot 34)$ & 0.015 & $1 \cdot 58(1.01-2 \cdot 49)$ & 0.046 & $1 \cdot 29(0 \cdot 62-2 \cdot 71)$ & 0.492 & $2.01(1.02-3.97)$ & 0.044 \\
\hline $\begin{array}{l}\text { Acid-suppressive } \\
\text { medication }\end{array}$ & $1.93(1.52-2 \cdot 44)$ & $<0.0001$ & $1.53(1.16-2 \cdot 03)$ & 0.003 & $2 \cdot 83(1.70-4 \cdot 67)$ & $<0.0001$ & $2.61(1.49-4.57)$ & 0.001 \\
\hline Analgesics & $0.95(0.75-1.20)$ & 0.654 & $0.94(0.71-1.24)$ & 0.653 & $1.43(0.85-2.41)$ & 0.177 & $0.81(0.45-1.44)$ & 0.47 \\
\hline Antidepressant & $0.63(0.47-0.83)$ & 0.001 & $0.61(0.44-0.85)$ & 0.003 & $0.57(0.31-1.03)$ & 0.062 & $0.72(0.39-1 \cdot 33)$ & 0.299 \\
\hline
\end{tabular}

$A O R=$ adjusted odds ratio. $P D S=$ postprandial distress syndrome. $E P S=$ =epigastric pain syndrome. $P H Q-12=P a t i e n t$ Health Questionnaire-12 somatic symptom scale. SF8-PCS=short form-8 quality-of-life form-physical component score. SF8-MCS=SF8-mental component score. FGIDs=functional gastrointestinal disorders.

Table 3: Multivariate analysis evaluating the associations of participants with Rome IV functional dyspepsia and subtypes in comparison with individuals with no dyspepsia

psychotropics (tricyclic antidepressants, antipsychotics, or both, but not selective serotonin reuptake inhibitors) to be more effective $t$ han $p$ lacebo $f$ or $t$ he $t$ reatment o $f$ functional dyspepsia (number needed to treat [NNT] 6), there is a paucity of high-quality randomised controlled trials, and which subtype of functional dyspepsia will benefit from psychotropics has not been elucidated. ${ }^{44}$ This is pertinent given that other treatment methods for functional dyspepsia are either restricted through concerns about their safety profile (ie, $\mathrm{p}$ rokinetics) o $\mathrm{r}$ show modest benefit o nly (ie, p roton-pump i nhibitors [NNT 13] and H pylori eradication [NNT 14]). ${ }^{45,46}$

Our findings might have important clinical and research implications as they bring into contention the published American College of Gastroenterology (ACG) and Canadian Association of Gastroenterology (CAG) joint guidelines on the management of dyspepsia. ${ }^{47}$
The guidelines define dyspepsia as epigastric pain, and provide step-wise treatment recommendations including H pylori eradication, proton-pump inhibitors, tricylic antidepressants, and prokinetics. ${ }^{47}$ However, our study shows that across both countries, and in the UK, the predominant Rome IV functional dyspepsia subtype is by far postprandial distress syndrome (about 60\%), which could imply that the ACG and CAG guidelines on dyspepsia might not necessarily be applicable to most individuals affected by functional dyspepsia within these countries. It then becomes unclear how such individuals with postprandial distress syndrome would be diagnosed or treated. Therapies for functional dyspepsia are modest at best and largely unsatisfactory for postprandial distress syndrome. For example, proton-pump inhibitors are ineffective for postprandial distress syndrome, and their unnecessary prescription would incur considerable cost 
and potential side-effects. ${ }^{48}$ Although $H$ pylori eradication can help with postprandial distress syndrome, ${ }^{49}$ as with epigastric pain syndrome, the prevalence of $H$ pylori is on the decline among white Americans, which will conceivably render this approach less common..$^{50,51}$ The role of antidepressants warrants further investigation within the functional dyspepsia subtypes, particularly for postprandial distress syndrome. Treatment with prokinetics is limited due to its significant adverse event profile. There is promising data for acotiamide, a first-in-class acetylcholinesterase inhibitor and muscarinic receptor agonist, to improve the symptoms of postprandial distress syndrome through accelerating gastric emptying and improving gastric accommodation; however, these findings are from a Japanese study, and are awaited in other populations. ${ }^{52}$

In summary, there is currently little in the way of optimism for patients with functional dyspepsia in North America and Europe, especially those with postprandial distress syndrome, who represent the majority, because of an absence of effective therapeutic options. This is reflected by a questionnaire that calculated the mean cost of dyspepsia to patients as US\$700 per year, ${ }^{6}$ and a study of the burden of gastrointestinal illness from the USA that reported almost two million physician visits in the year 2009 for dyspepsia. ${ }^{53}$ The economic and societal burden generated by individuals with functional dyspepsia was conservatively estimated to cost the USA at least $\$ 18.4$ billion during $2009 .{ }^{6}$ Furthermore, individuals with functional dyspepsia are commonly under diagnosed in clinic, with only $12.5 \%$ of those with functional dyspepsia receiving the diagnosis. ${ }^{54}$ Instead, they are more likely to be classed as having gastro-oesophageal reflux disease, even if acid-suppressive therapy is unsuccessful..$^{54}$ It is therefore imperative to raise awareness of the epidemiological scale of Rome IV functional dyspepsia and recognition of its subtypes, to aid future clinical service and research provision, and hopefully lead to guidelines and effective therapy addressing all functional dyspepsia subtypes.

The strength of our study is that it is the first to sample the prevalence of functional dyspepsia, using the Rome IV criteria, ${ }^{21}$ across a large sample of adults from three English-speaking countries, using the same systematic methodological process across countries. Moreover, we provide a comprehensive perspective on multiple characteristics seen in Rome IV functional dyspepsia and how they are independently associated with individual subtypes. It is logical to assume that, had our study been performed across multiple communities using the Rome III criteria, the prevalence would be expected to be similar to Rome IV, given that the main diagnostic criteria have remained unaltered by contrast with the historical Rome I or II iterations. ${ }^{4,21,55}$ However, no study has simultaneously evaluated the prevalence of functional dyspepsia across multiple communities using any of the previous Rome criteria. ${ }^{2}$ Instead, they have been limited to evaluations within single communities, which on pooling within their respective criteria have shown significant heterogeneity, irrespective of their geographical location. ${ }^{2}$ Hence, our study fills a void in the literature since the introduction of the Rome criteria almost 30 years ago.

The main limitation of our study is that the diagnosis of functional dyspepsia was based on fulfilling symptom-based criteria and was not subsequently confirmed by clinical evaluation or endoscopy. Our study could not feasibly perform endoscopy due to its widespread dissemination and internet basis. Furthermore, we did not ask participants whether they had previously undergone an endoscopy, because descriptive responses, particularly for endoscopic findings, would not be reliable and could not be verified. Hence, our participants can be termed as having uninvestigated dyspepsia. Nevertheless, we would argue that performing population-based endoscopic studies for epidemiological studies of dyspepsia within the North American and European population might no longer be necessary in view of its low diagnostic yield. In fact, three large population-based endoscopic studies ${ }^{9,26,27}$ have shown that most individuals with dyspepsia do not have organic pathology at endoscopy $(>70 \%)$ and so will be diagnosed with functional dyspepsia, which supports our assumption. Furthermore, in those in whom organic pathology is noted, oesophagitis is among the most common finding (about $9 \%$ of all dyspepsia cases, therefore approximately a third of organic dyspepsia cases), ${ }^{26}$ but its role in causing dyspeptic symptoms is doubtful given that similar prevalence rates are seen in asymptomatic controls. ${ }^{56}$ The presence of peptic ulcer disease has been reported in about $9 \%$ and gastric cancer in up to about $2 \%$ in patients with dyspepsia. ${ }^{26,27}$ In this regard, the joint ACG and CAG guidelines acknowledge that gastric cancer is rare in individuals with dyspepsia presenting at younger than 60 years and thus do not routinely recommend upper gastrointestinal endoscopy as a first-line management plan in this group. ${ }^{47}$ Rather, they suggest to test and treat for $H$ pylori, followed by treatment algorithms outlined for functional dyspepsia. ${ }^{47}$ The UK National Institute for Health and Care Excellence has a similar policy of not recommending upper gastrointestinal endoscopy as a routine first-line test in those with dyspepsia younger than 55 years without alarm symptoms. ${ }^{57}$ Furthermore, our dataset is limited to three English-speaking countries and does not cover the epidemiology of functional dyspepsia globally. Our results therefore cannot be generalised to all countries. However, a global functional gastrointestinal disorder epidemiology study with comparable methodology is now underway and will help to address these issues. Another limitation of our study is that we sampled at a cross-sectional timepoint; however, longitudinal follow-up studies have shown that the prevalence of functional dyspepsia remains relatively stable over time. ${ }^{33}$ 
Finally, there are advantages and disadvantages to doing population-based surveys irrespective of whether this is through postal, face-to-face, telephone, or internet-based methods; these have been extensively discussed elsewhere for our dataset. ${ }^{18}$ This includes, on the one hand, the absence of a denominator, and on the other hand, the ability to reach large populations using cost-effective a nd $\mathrm{t}$ ime-effective me thods, performing quota-based sampling (for age, sex, and education) to obtain demographically balanced and nationally representative participants, building in quality assurance methods to ensure no missing data and eliminate inconsistent responders, and introducing the survey as one of general health without mention of gastrointestinal emphasis. ${ }^{18}$ Hence, despite methodological concerns regarding potential selection bias, we believe that necessary measures were undertaken to counteract this when possible and provide a representative epidemiological profile across the three countries using a systematic and consistent methodology ${ }^{18}$ Another issue worth noting is that the prevalence of functional dyspepsia has been recorded to be lower with internet surveys than with other methods of data collection. ${ }^{2}$ However, internet surveys in this field have been limited to a few studies and not subjected to broad historical definitions $f$ or functional dyspepsia, which are generally associated with higher prevalence rates. ${ }^{2}$ It has also been speculated that personal approaches to collecting data, such as face-to-face or telephone surveys, might overestimate the prevalence of dyspepsia compared with more impersonal methods such as internet-based surveys. ${ }^{2}$

In conclusion, approximately $10 \%$ of the adult population from the USA, Canada, and the UK fulfil symptom-based criteria for Rome IV functional dyspepsia, and these individuals incur considerable health impairment. The factors associated with functional dyspepsia differ a ccordingt os ubtype, s uggesting different $\mathrm{p}$ athophysiological $\mathrm{p}$ rocesses o $\mathrm{r}$ i nfluences. These findings a re 1 ikely to i ncrease a wareness of the epidemiological scale of functional dyspepsia in the general population and its detrimental effect on general health, and will hopefully aid clinical service and guide research planning, particularly in determining the role of antidepressants.

\section{Contributors}

OSP and WEW contributed to the study design, questionnaire development, execution of survey, data processing, and manuscript editing. ADS contributed to the study design, questionnaire development, and manuscript editing. HT contributed to interpretation of data, and manuscript editing. MS contributed to study design, interpretation of data and manuscript editing. IA analysed the data and drafted the manuscript. All authors reviewed and approved the final version of the manuscript.

\section{Declaration of interests}

MS has received unrestricted research grants from Danone and Ferring Pharmaceuticals; and has served as a consultant or advisory board member for AstraZeneca, Danone, Nestlé, Almirall, Allergan, Albireo, Glycom, and Shire; and as a speaker for Tillotts, Menarini, Takeda, Shire, Allergan, and Almirall. HT has served as a consultant or advisory board member for Almirall and Shire. OSP has received salary support from research grants from Takeda Pharmaceuticals and Salix Pharmaceuticals, from a consulting agreement with Ironwood Pharmaceuticals, and an educational grant provided by Takeda Pharmaceuticals; and received a speaker honorarium in an educational programme supported by Ironwood Pharmaceuticals and Takeda Pharmaceuticals. WEW received research grants from Takeda, Ironwood, Salix, and the Rome Foundation; served as a consultant to Biomerica USA, Ono Pharmaceuticals and Ferring; and received unrestricted educational grants from Takeda and Ferring. IA and ADS declare no competing interests.

\section{Acknowledgments}

We thank the Rome Foundation, US National Institute of Diabetes and Digestive and Kidney Diseases (grant RO1 DK31369), the Swedish Medical Research Council (grants 13409, 21691 and 21692), AFA Insurance, an unrestricted grant from Ferring Pharmaceuticals, and the Faculty of Medicine, University of Gothenburg for helping to fund this study.

References

1 Talley NJ, Ford AC. Functional dyspepsia. N Engl J Med 2015; 373: 1853-63.

2 Ford AC, Marwaha A, Sood R, Moayyedi P. Global prevalence of, and risk factors for, uninvestigated dyspepsia: a meta-analysis. Gut 2015; 64: 1049-57.

3 Ford AC, Marwaha A, Lim A, Moayyedi P. What is the prevalence of clinically significant endoscopic findings in subjects with dyspepsia? Systematic review and meta-analysis. Clin Gastroenterol Hepatol 2010; 8: 830-37.

4 Tack J, Talley NJ, Camilleri M, et al. Functional gastroduodenal disorders. Gastroenterology 2006; 130: 1466-79.

5 Brook RA, Kleinman NL, Choung RS, Melkonian AK, Smeeding JE, Talley NJ. Functional dyspepsia impacts absenteeism and direct and indirect costs. Clin Gastroenterol Hepatol 2010; 8: 498-503.

6 Lacy BE, Weiser KT, Kennedy AT, Crowell MD, Talley NJ. Functional dyspepsia: the economic impact to patients. Aliment Pharmacol Ther 2013; 38: 170-77.

7 Fang YJ, Liou JM, Chen CC, et al. Distinct aetiopathogenesis in subgroups of functional dyspepsia according to the Rome III criteria. Gut 2015; 64: 1517-28.

8 Hsu YC, Liou JM, Liao SC, et al. Psychopathology and personality trait in subgroups of functional dyspepsia based on Rome III criteria. Am J Gastroenterol 2009; 104: 2534-42.

9 Aro P, Talley NJ, Ronkainen J, et al. Anxiety is associated with uninvestigated and functional dyspepsia (Rome III criteria) in a Swedish population-based study. Gastroenterology 2009; 137: 94-100.

10 Gracie DJ, Bercik P, Morgan DG, et al. No increase in prevalence of somatization in functional vs organic dyspepsia: a cross-sectional survey. Neurogastroenterol Motil. 2015; 27: 1024-31.

11 Aro P, Talley NJ, Agréus L, et al. Functional dyspepsia impairs quality of life in the adult population. Aliment Pharmacol Ther 2011; 33: $1215-24$

12 Ford AC, Marwaha A, Lim A, Moayyedi P. Systematic review and meta-analysis of the prevalence of irritable bowel syndrome in individuals with dyspepsia. Clin Gastroenterol Hepatol 2010; 8: 401-09.

13 Locke GR, Zinsmeister AR, Fett SL, Melton LJ, Talley NJ. Overlap of gastrointestinal symptom complexes in a US community. Neurogastroenterol Motil 2005; 17: 29-34.

14 Mahadeva S, Ford AC. Clinical and epidemiological differences in functional dyspepsia between the East and the West. Neurogastroenterol Motil 2016; 28: 167-74.

15 Drossman DA, Hasler WL. Rome IV-Functional GI Disorders: disorders of gut-brain interaction. Gastroenterology 2016; 150: 1257-61.

16 Drossman DA. Functional gastrointestinal disorders: history, pathophysiology, clinical features and Rome IV. Gastroenterology 2016; 150: 1262-79.

17 Palsson OS, Whitehead WE, van Tilburg MA, et al. Rome IV diagnostic questionnaires and tables for investigators and clinicians. Gastroenterology 2016; 150: 1481-91.

18 Aziz I, Palsson OS, Törnblom H, Sperber AD, Whitehead WE, Simrén M. The prevalence and impact of overlapping Rome IV-diagnosed functional gastrointestinal disorders on somatization, quality of life, and healthcare utilization: a cross-sectional general population study in three countries. Am J Gastroenterol 2018; 113: 86-96. 
19 Spiller RC, Humes DJ, Campbell E, et al. The Patient Health Questionnaire 12 Somatic Symptom scale as a predictor of symptom severity and consulting behaviour in patients with irritable bowel syndrome and symptomatic diverticular disease. Aliment Pharmacol Ther 2010; 32: 811-20.

20 Ware JE, Kosinski M, Dewey JE, Gandek B. How to score and interpret single-item health status measures: a manual for users of the SF-8 health survey. Lincoln, RI, USA: Quality Metric Incorporated, 2001.

21 Stanghellini V, Chan FK, Hasler WL, et al. Gastroduodenal disorders. Gastroenterology 2016; 150: 1380-92.

22 Aziz Q, Fass R, Gyawali CP, Miwa H, Pandolfino JE, Zerbib F. Functional esophageal disorders. Gastroenterology 2016; 150: 1368-79.

23 Lacy BE, Mearin F, Chang L, et al. Bowel disorders. Gastroenterology 2016; 150: 1393-407.

24 Talley NJ. Functional dyspepsia: advances in diagnosis and therapy. Gut Liver 2017; 11: 349-57.

25 Jones MP, Walker MM, Ford AC, Talley NJ. The overlap of atopy and functional gastrointestinal disorders among 23,471 patients in primary care. Aliment Pharmacol Ther 2014; 40: 382-91.

26 Zagari RM, Law GR, Fuccio L, et al. Epidemiology of functional dyspepsia and subgroups in the Italian general population: an endoscopic study. Gastroenterology 2010; 138: 1302-11.

27 Bernersen B, Johnsen R, Straume B. Non-ulcer dyspepsia and peptic ulcer: the distribution in a population and their relation to risk factors. Gut 1996; 38: 822-25.

28 Wang A, Liao X, Xiong L, et al. The clinical overlap between functional dyspepsia and irritable bowel syndrome based on Rome III criteria. BMC Gastroenterol 2008; 8: 43.

29 van Kerkhoven LA, Laheij RJ, Meineche-Schmidt V, Veldhuyzen-van Zanten SJ, de Wit NJ, Jansen JB. Functional dyspepsia: not all roads seem to lead to rome. J Clin Gastroenterol 2009; 43: 118-22.

30 Carbone F, Holvoet L, Tack J. Rome III functional dyspepsia subdivision in PDS and EPS: recognizing postprandial symptoms reduces overlap. Neurogastroenterol Motil 2015; 27: 1069-74.

31 Ford AC, Forman D, Bailey AG, Cook MB, Axon AT, Moayyedi P. Who consults with dyspepsia? Results from a longitudinal 10-yr follow-up study. Am J Gastroenterol 2007; 102: 957-65.

32 van Bommel MJ, Numans ME, de Wit NJ, Stalman WA. Consultations and referrals for dyspepsia in general practice-a one year database survey. Postgrad Med J 2001; 77: 514-18.

33 Ford AC, Forman D, Bailey AG, Axon AT, Moayyedi P. Initial poor quality of life and new onset of dyspepsia: results from a longitudinal 10-year follow-up study. Gut 2007; 56: 321-27.

34 Vanheel H, Carbone F, Valvekens L, et al. Pathophysiological abnormalities in functional dyspepsia subgroups according to the Rome III criteria. Am J Gastroenterol 2017; 112: 132-40.

35 Shindo T, Futagami S, Hiratsuka T, et al. Comparison of gastric emptying and plasma ghrelin levels in patients with functional dyspepsia and non-erosive reflux disease. Digestion 2009; 79: 65-72.

36 Haag S, Senf W, Tagay S, et al. Is there any association between disturbed gastrointestinal visceromotor and sensory function and impaired quality of life in functional dyspepsia? Neurogastroenterol Motil 2010; 22: 262-79.

37 Di Stefano M, Miceli E, Tana P, et al. Fasting and postprandial gastric sensorimotor activity in functional dyspepsia: postprandial distress vs. epigastric pain syndrome. Am J Gastroenterol 2014; 109: 1631-39.

38 Ochi M, Tominaga K, Tanaka F, et al. Clinical classification of subgroups according to the Rome III criteria cannot be used to distinguish the associated respective pathophysiology in Japanese patients with functional dyspepsia. Intern Med 2013; 52: 1289-93.
39 Bouras EP, Talley NJ, Camilleri M, et al. Effects of amitriptyline on gastric sensorimotor function and postprandial symptoms in healthy individuals: a randomized, double-blind, placebo-controlled trial. Am J Gastroenterol 2008; 103: 2043-50.

40 Tack J, Janssen P, Masaoka T, Farré R, Van Oudenhove L. Efficacy of buspirone, a fundus-relaxing drug, in patients with functional dyspepsia. Clin Gastroenterol Hepatol 2012; 10: 1239-45.

41 Janssen P, Van Oudenhove L, Casteels C, Vos R, Verbeke K, Tack J. The effects of acute citalopram dosing on gastric motor function and nutrient tolerance in healthy volunteers. Aliment Pharmacol Ther 2011; 33: 395-402.

42 Arienti V, Corazza GR, Sorge M, et al. The effects of levosulpiride on gastric and gall-bladder emptying in functional dyspepsia. Aliment Pharmacol Ther 1994; 8: 631-38.

43 Tack J, Broekaert D, Coulie B, Fischler B, Janssens J. Influence of the selective serotonin re-uptake inhibitor, paroxetine, on gastric sensorimotor function in humans. Aliment Pharmacol Ther 2003; 17: 603-08.

44 Ford AC, Luthra P, Tack J, Boeckxstaens GE, Moayyedi P, Talley NJ. Efficacy of psychotropic drugs in functional dyspepsia: systematic review and meta-analysis. Gut 2017; 66: 411-20.

45 Pinto-Sanchez MI, Yuan Y, Bercik P, Moayyedi P. Proton pump inhibitors for functional dyspepsia. Cochrane Database Syst Rev 2017; 3: CD011194.

46 Moayyedi P, Soo S, Deeks J, et al. Eradication of Helicobacter pylori for non-ulcer dyspepsia. Cochrane Database Syst Rev 2006; 2: CD002096.

47 Moayyedi PM, Lacy BE, Andrews CN, Enns RA, Howden CW, Vakil N. ACG and CAG clinical guideline: management of dyspepsia. Am J Gastroenterol 2017; 112: 988-1013.

48 Moayyedi P, Delaney BC, Vakil N, Forman D, Talley NJ. The efficacy of proton pump inhibitors in nonulcer dyspepsia: a systematic review and economic analysis. Gastroenterology 2004; 127: 1329-37.

49 Suzuki H, Moayyedi P. Helicobacter pylori infection in functional dyspepsia. Nat Rev Gastroenterol Hepatol 2013; 10: 168-74.

50 Eusebi LH, Zagari RM, Bazzoli F. Epidemiology of Helicobacter pylori infection. Helicobacter 2014; 19 (suppl 1): 1-5.

51 Chey WD, Leontiadis GI, Howden CW, Moss SF. ACG Clinical Guideline: treatment of Helicobacter pylori infection. Am J Gastroenterol 2017; 112: 212-39.

52 Matsueda K, Hongo M, Tack J, Saito Y, Kato H. A placebo-controlled trial of acotiamide for meal-related symptoms of functional dyspepsia. Gut 2012; 61: 821-28.

53 Peery AF, Dellon ES, Lund J, et al. Burden of gastrointestinal disease in the United States: 2012 update. Gastroenterology 2012; 143: 1179-87.

54 Pleyer C, Bittner H, Locke GR, et al. Overdiagnosis of gastro-esophageal reflux disease and underdiagnosis of functional dyspepsia in a USA community. Neurogastroenterol Motil 2014; 26: $1163-71$.

55 Talley NJ, Stanghellini V, Heading RC, Koch KL, Malagelada JR, Tytgat GN. Functional gastroduodenal disorders. Gut 1999; 45 (suppl 2): 1137-42.

56 Zagari RM, Law GR, Fuccio L, Pozzato P, Forman D, Bazzoli F. Dyspeptic symptoms and endoscopic findings in the community: the Loiano-Monghidoro study. Am J Gastroenterol 2010; 105: 565-71.

57 National Institute of Health and Care Excellence. Referral guidelines for suspected cancer. June, 2005. https://www.nice.org. uk/guidance/cg27 (accessed Sept 1, 2017). 